\title{
Classification and Recognition of Operators' Mental Load Under Repetitive High-precision Tasks Based on BP Neural Network
}

\author{
Wenmin Han ${ }^{1, *}$, Huimin Qiang ${ }^{1}$, Peiyao $\mathrm{Li}^{1}$ \\ ${ }^{1}$ School of Economics and Management, Jiangsu University of Science and Technology, Zhenjiang, Jiangsu, China \\ ${ }^{*}$ Corresponding author. Email:769691107@qq.com
}

\begin{abstract}
In order to identify the mental load of operators under repetitive high-precision tasks effectively, 36 subjects were recruited in this study. The fine tasks on the electronic assembly line were simulated in the laboratory, and operators' behavioural performance (completion time, error rate) and the changes of Oxyhaemoglobin $\left(\mathrm{O}_{2} \mathrm{Hb}\right)$ in prefrontal channels 2, 8, 12, 17, 20 and 21 of the brain were characteristic factors for mental load recognition. A model of recognition of mental load state of operators based on BP neural network was constructed and the fatigue state of operators was divided into four levels. Finally, the recognition rate of the mental load state of operators was $86.81 \%$ by combining the experimental data. The combination of behavioural performance indicators and physiological measurement indicators can effectively identify mental work state of operators, which provides a new idea for classification and recognition of mental load state of operators under repetitive high-precision operation tasks, and provides a reference for the establishment of effective labour organization.
\end{abstract}

Keywords: Repetitive high-precision tasks, mental load, Oxyhaemoglobin $\left(\mathrm{O}_{2} \mathrm{Hb}\right), \mathrm{BP}$ neural network

\section{INTRODUCTION}

With highly mechanized and automated manufacturing workshops of today, although modern equipment can complete the majority of manual operations, numerous repetitive and high-precision manual assembly operations, persist, including precision assembly of armature parts [1] and precision parts assembly with small tolerance range [2]. While the automated machinery requires operators to maintain high attention and invest significant cognitive resources; it also requires them to possess better finger control ability to ensure precise pickup, movement, and manipulation of objects from a place [3,4], thereby resulting in a significant upsurge in the mental and physical load of operators during the task.

To date, many studies have investigated the physical load of operators in repetitive high-precision tasks. Reportedly, high repeatability and precision tasks decrease the variability of operators [5], thereby increasing of the physical load of operators. However, manufacturing enterprises upgraded operation tools and auxiliary equipment to decrease static operation and reduce the physical load of operators [6]. Nevertheless, in repetitive high-precision tasks, the mental load remains a concern that cannot be overlooked. Of note, the excessive mental load could decrease production efficiency and reaction sensitivity, resulting in human error [7]. Therefore, it is necessary to predict the mental load of operators in the process of repetitive highprecision tasks.

In recent years, neural networks are mostly used in data prediction and state recognition. Among them, BP neural network is the most widely used neural network [8]. Mingzhou Liu [9] used BP neural network to establish a fatigue prediction model based on drivers' facial and hand features. Liuye Yao [10] used BP neural network to predict the fatigue caused by stereo vision. BP neural network has the function of nonlinear mapping, and on this basis, it can learn, organize, and adapt itself [11]. Therefore, this paper used a three-layer BP neural network to establish a predictive model of operators' mental load.

Compared with previous studies, this paper focused on the mental load of operators under repetitive highprecision tasks, and used functional near - infrared spectroscopy (fNIRS) to measure the changes of $\mathrm{O}_{2} \mathrm{Hb}$ in 
the prefrontal lobe of operators during tasks, which is real-time and objective. At the same time, based on BP neural network, the task performance indicators and physiological data indicators of operators were combined to build a recognition model of mental load state of operators under repetitive and high-precision tasks. In this paper, the participants were required to complete the jack experiment with high repeatability and high precision in the laboratory, which was similar to the assembly of precision instrument parts in the electronic factory, with consistency and typicality. In the process of experiment, the operators' jack time, jack error rate, and the changes of $\mathrm{O}_{2} \mathrm{Hb}$ in the prefrontal lobe were recorded. Combined with BP neural network, the repetitive and high-precision tasks recognition model was constructed to identify mental load state of operators.

\section{MATERIAL AND METHODS}

\subsection{Participants}

Before the formal experiment, we enrolled 36 participants aged 21-26 (23.81 \pm 1.41$)$. All participants had normal or corrected-to-normal vision, were righthanded, and had no physical disorder. In addition, all participants were asked to get sufficient sleep, not use drugs or drink alcohol, tea, or coffee, and refrain from strenuous upper body exercise or other fatiguing activities. Notably, all participants were informed about the objectives and conditions of the experiment, and written informed consent was obtained from all. This study was conducted in compliance with the principles and guidelines of the Declaration of Helsinki and all participants were assured of getting a certain reward.

\subsection{Experimental equipment}

During the experiment, Nikon cameras were used to record videos of participants completing repetitive highprecision tasks for subsequent behavioural data analysis. At the same time, the prefrontal cortex was monitored by fNIRS, and the change of $\mathrm{O}_{2} \mathrm{Hb}$ in each channel was recorded.

\subsection{Experimental task}

In this study, repetitive high-precision tasks on the assembly line of the electronic factory were designed to simulate the jack experiment. In the jack experiment, participants were to use their right hand to hold tweezers to insert 100 small needles into the holes with a $1.5-\mathrm{mm}$ diameter on the metal plate. During the task, participants had to follow the jack sequence from left to right, from top to bottom, and each needle had to be inserted into the bottom of the small hole, and participants did not rest during the experiment. If participants dropped a needle intentionally or unintentionally, they had to pick up the small needle with tweezers and insert it into the corresponding holes. Furthermore, participants were asked to try their best to minimize the total time of completion. And, fNIRS was used to monitor the prefrontal cortex of the participants' brain in real time, and participants were not allowed to talk or do other unrelated matters.

\section{ESTABLISHMENT OF MENTAL LOAD RECOGNITION MODEL}

\subsection{Determination of mental load identification factors}

Previous studies have shown that the performance of operators can effectively reflect the mental load of operators [12]. Therefore, this paper analyzed the validity of the objective evaluation index of mental load in the process of tasks, and found that the two objective evaluation indexes of jack time and jack error rate had good discriminative validity. At the same time, the fNIRS data of 12 prefrontal cortex channels were analyzed. The results showed that prefrontal cortex channels $2,8,12$, 17, 20 and 21 was significantly activated during the experiment, which affected the mental load of operators during the experiment. Channel 2 corresponds to the sub frontal cortex, channels 8,12 to the medial prefrontal cortex, and channels 17, 20 and 21 to the dorsolateral prefrontal cortex. The sub frontal cortex is the main emotion control area in the prefrontal cortex, which participates in many cognitive and emotional regulation functions in the jack experiment; the medial prefrontal cortex mainly participates in cognitive functions such as working memory, which is responsible for temporarily storing the memory content; the dorsolateral prefrontal cortex is an important area associated with central execution domain, which mainly monitors tasks and allocates the consumption of cognitive resources, is highly sensitive to changes in mental load. Therefore, the eight indicators of jack time, jack error rate and $\mathrm{O}_{2} \mathrm{Hb}$ changes of prefrontal channel 2,8,12,17,20 and 21 could effectively evaluate the mental load of participants in the repetitive high-precision tasks.

\subsection{Extract the principal components}

The feasibility test was performed on 36 participants and a total of 144 sets of data in four experimental stages. The KMO statistic was $0.768>0.600$, and the Bartlett sphere test was statistically significant $(\mathrm{P}=0.000<0.010)$. The characteristic roots of the two principal components F1 and F2 were 3.327 and 1.291 respectively. The variance contribution rates of $\mathrm{F} 1$ and $\mathrm{F} 2$ were $41.584 \%$ and $28.274 \%$. Therefore, this paper selected two principal components to comprehensively identify and evaluate the mental load of operators. 


\subsection{Comprehensive evaluation model of mental load}

Before the comprehensive evaluation of the mental load of operators, it was necessary to determine the primary weight model of the mental load evaluation, as shown in Equation (1).

$\left\{\begin{array}{c}F_{1}=a_{11} x_{1}+a_{21} x_{2}+\cdots+a_{k 1} x_{k} \\ F_{2}=a_{12} x_{1}+a_{22} x_{2}+\cdots+a_{k 2} x_{k} \\ \vdots \\ F_{p}=a_{1 p} x_{1}+a_{2 p} x_{2}+\cdots+a_{k p} x_{k}\end{array}\right.$

In Equation (1), $F_{p}$ represents the obtained $p$-th principal component; $a_{i j}$ represents the coefficient in the primary weight model, and the specific calculation formula was shown as Equation (2):

$a_{i j}=\frac{\theta_{i j}}{\sqrt{\lambda_{j}}}(i=1,2, \cdots, k, j=1,2, \cdots, p)$

In Equation (2), $\theta_{i j}$ represents the initial factor load of the $j$-th principal component corresponding to the $i$-th index; $\lambda_{j}$ represents the characteristic root of the $j$-th principal component, and the primary weight model was calculated. Then, the weighted average of the variance contribution rate of two indicators in the principal component analysis was used to obtain the weight of each indicator. Finally, normalize the index weights to obtain the normalized comprehensive index system model of mental load was as Equation (3):

$F=-0.067 x_{1}+0.059 x_{2}+0.237 x_{3}+0.166 x_{4}+$ $0.200 x_{5}+0.120 x_{6}+0.129 x_{7}+0.156 x_{8}$

After obtaining the comprehensive index system model, the time index, error rate index and $\mathrm{O}_{2} \mathrm{Hb}$ index of 144 sets of data of 36 subjects in the four experimental stages were normalized and brought into the mental load comprehensive index evaluation model, and the mental load values of four experimental stages were obtained, as shown in Figure 1.

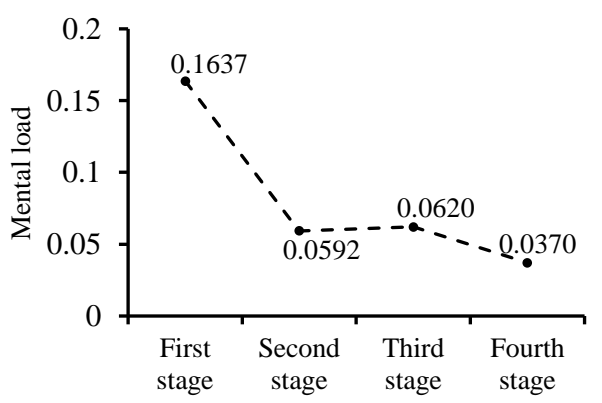

Figure 1 Diagram of the average mental load.

\section{RECOGNITION MODEL OF MENTAL LOAD STATE BASED ON BP NEURAL NETWORK}

\subsection{Model establishment}

The mental load of operators in four experimental stages was analysed. The results showed that there were significant differences among the four levels of mental load. Therefore, this paper divided the mental load of operators under repetitive high-precision tasks into four levels, and the four mental load levels were used as the output items of the network to construct the BP neural network model of operators' mental load state under repetitive high-precision tasks. BP neural network does not need to construct equations in advance to indicate the relationship between independent variables and dependent variables. It can independently learn the mapping mode from input variables to output variables during the simulation process [13].

Based on the establishment of the BP neural network recognition model of operators' mental load under repetitive high-precision tasks, the following indicators of the network model needed to be determined: (1) Number of input neurons: 8; (2) Number of network layers: 3 layers; (3) Number of hidden layer neurons: 9; (4) Number of output neurons: 4; (5) Transfer function selection: tansig function; (6) Training function selection: trainlm function; (7) Expected error determination: 0.0001 .

\subsection{Training and testing of the network model}

In this paper, about $3 / 4$ of the 36 participants, that is, 104 groups of data in four experimental stages were collected as training samples, and the 8 indexes of 26 , participants were input into the BP neural network mental load prediction model, which was trained by MATLAB, as shown in Figure 2.

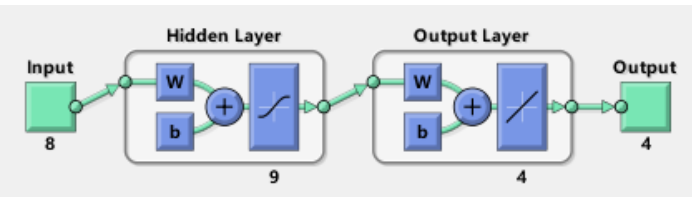

Figure 2 Structure of BP neural network model.

Before the neural network training and testing, the data should be normalized in the neural network, with 1000 as the maximum number of iterations and the learning rate of 0.01 . Using MATLAB programming, the samples were trained and tested for many times. Based on the above steps, samples' mental load level was predicted, as shown in Figure 3 and Figure 4, the recognition rate of training samples' mental load level reached $89.43 \%$, the recognition rate of testing samples' mental load level reached $80 \%$, and the recognition rate of total samples' mental load level reached $86.81 \%$. It can 
be considered that the BP neural network has basically achieved the expected effect, and can be used to objectively identify the mental load state of operators under repetitive and high-precision tasks, which overcame the subjectivity of using scale as the evaluation index of mental load of operators in the past.

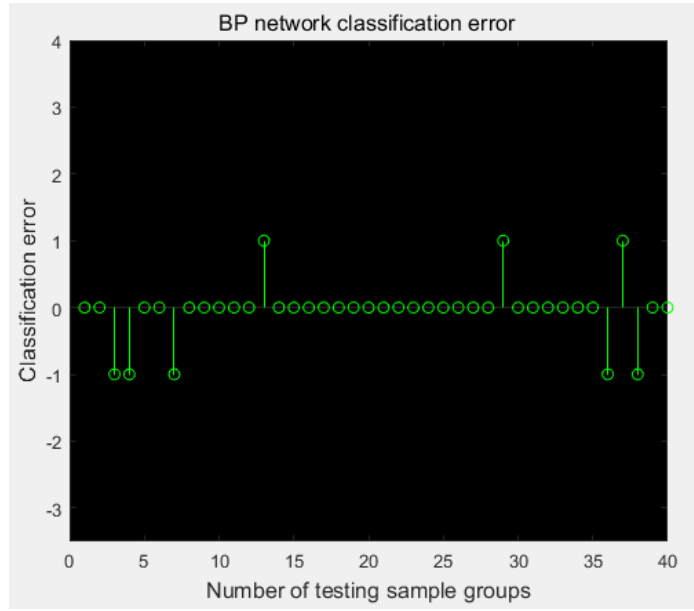

Figure 3 Error map of testing set.

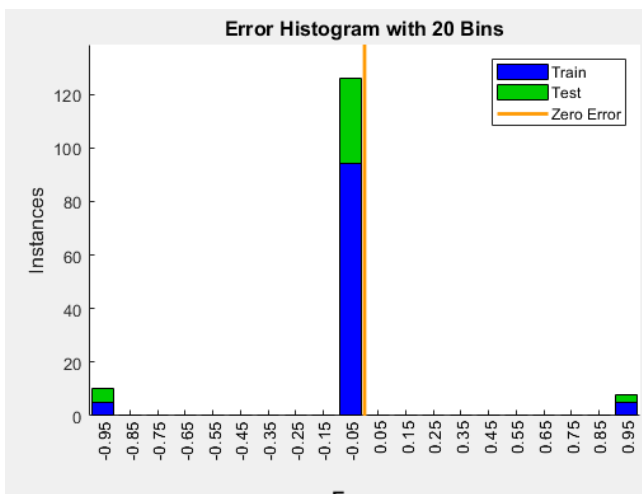

Figure 4 Error distribution histogram of training set and testing set.

\section{CONCLUSION}

(1) The main component was used as the characteristic parameter of BP neural network recognition model by principal component analysis. The objectivity of operators' mental load level recognition was further improved, and the subjective factor involved in the former subjective scale evaluation of legal person as parameter factor was overcame.

(2) The model of mental load state prediction based on BP neural network was constructed based on the recognition factors of operators' behavioral performance and $\mathrm{O}_{2} \mathrm{Hb}$ concentration in six channels of prefrontal lobe of brain under repetitive high-precision tasks. The model was verified by experimental data. The recognition rate of mental load reached $86.81 \%$, which proved the accuracy and effectiveness of the recognition model.

(3) Under repetitive and high-precision tasks, the mental load of operators is closely related to the behavioral performance. The enterprise managers should always pay attention to whether the operators are overloaded or not, and establish a reasonable rest system based on the identification model of the mental load state to reduce the mental load, stabilize the working efficiency and realize high efficiency production.

\section{AUTHORS' CONTRIBUTIONS}

W. Han and H. Qiang conceived of the study, designed the study, and analysed the data. All authors contributed to the writing and revisions.

\section{ACKNOWLEDGMENTS}

This work was supported by the National Natural Science Foundation of China (NEFC) [71271105].

\section{REFERENCES}

[1] Wang X., Lou Z., Wang X., Wang Y., Hao X. \& Wang Z. "Automatic press-fit assembly of small precision interference fitting parts: armature of electro-hydraulic servo valve", Assembly Automation, 2019, Vol. 39 No. 5, pp. 986-998.

[2] Dong II Park, Hwisu Kim, Chanhun Park, Taeyong Choi,Jongwoo Park, \& Byungin Kim. Passive compliant module with the displacement measurement sensor and its application for automatic assembly[J]. IFAC PapersOnLine, 2018, 51(22). DOI: https://doi.org/ 10.1016/j.ifacol. 2018.11.522.

[3] Noor S., Shohan J. A., \& Waresi M. S., et al. Real time hand movement controlled robotic arm for risk prevention[C]. 2017 IEEE Region 10 Humanitarian Technology Conference (R10-HTC).

[4] CNOEMPhone. A simple review of chinese cellular phone manufacturing industry chain [Http://cnoemphone.net/blog/a-simple-review-ofchinese-cellular-phone-manufacturing-industrychain]. 2016.

[5] Srinivasan D., Mathiassen S. E., Samani A., \& Madeleine $\mathrm{P}$. The combined influence of task accuracy and pace on motor variability in a standardised repetitive precision task[J]. Ergonomics, 2015, 58(8), 1388-1397. DOI: https://doi.org/10.1080/00140139.2015.1005174.

[6] NØRregaard R. C. D., Rasmus H. P., \& Jagd S. M., et al. Improving work for the body - a participatory ergonomic intervention aiming at reducing physical exertion and musculoskeletal pain among childcare workers (the TOY-project): study protocol for a wait-list cluster-randomized controlled trial[J]. 
Trials, 2018, 19(1), 411. DOI: https://doi.org/10.1186/s13063-018-2788-z.

[7] Tom Eichele, Stefan Debener, \& Vince D. C. Prediction of human errors by maladaptive changes in event-related brain network[J]. Proceeding of the National Academy of Sciences, 2008, 105(16), 6173-6178.

DOI: https://doi.org/10.1073/pnas.0708965105

[8] Wen Xin. Intelligent fault diagnosis technology: MATLAB application[M]. Beijing University of Aeronautics and Astronautics Press, 2015.

[9] Mingzhou Liu, Qiannan Jiang, Jing Hu. Driver fatigue detection based on facial geometric features and hand motion features $[\mathrm{J}]$. Chinese Journal of Mechanical Engineering, 2018.

[10] Liuye Yao, Xiao Wang, Zhiyu Qian, et al. Research on stereo vision fatigue classification and prediction based on PCA and BP neural network[J]. Life Science Instruments, 2018, 16(06):30-36.

[11] So G J, Kim S H, Kim J Y. Evaluation Model of the Visual Fatigue on the 3D Stereoscopic Video[J]. Acta Chirurgica Scandinavica Supplementum, 2016, 325.

[12] Maja S, Chris B, Levendowski D J, et al. Modeling temporal sequences of cognitive state changes based on a combination of EEG-engagement, EEGworkload, and heart rate metrics[J]. Frontiers in Neuroscience, 2014, 8. DOI: https://doi.org /10.3389/fnins.2014.00342

[13] Yang Shuying, Zhang Hua. Pattern recognition and intelligent computing[M]. Electronic Industry Press, 2015. 